\title{
Common Fixed Point Theorems For Weakly Compatible Mappings In Generalisation Of Symmetric Spaces.
}

\author{
T.R.Vijayan, \\ Department of Mathematics, Pavai College of Technology, Namakkal - 637018, India
}

\begin{abstract}
The main purpose of this paper is to obtain common fixed point theorem for weakly compatible mappings in generalisation symmetric spaces and a Property (E.A) introduced in [M. Aamri, D. El Moutawakil, Some new common fixed point theorems under strict contractive conditions, J. Math. Anal. Appl. 270 (2002) 181-188]. Our theorem generalizes theorems Duran turkoglu and ishak altun, a common fixed point theorem for weakly compatible mappings in symmetric spaces satisfying Bol. Soc. Mat. Mexicana (3) Vol. 13, 2007.
\end{abstract}

Keywords and phrases: Common fixed point, weakly compatible mappings, symmetric space, and implicit relation.

\section{Introduction}

It is well known that the Banach contraction principle is a fundamental result in fixed point theory, which has been used and extended in many different directions. Hicks [5] established some common fixed point theorems in symmetric spaces and proved that very general probabilistic structures admit a compatible symmetric or semi-metric. Recall that a symmetric on a set $\mathrm{X}$ is a nonnegative real valued function $\mathrm{d}$ on $\mathrm{X} \times \mathrm{X}$ such that (i) $d(x, y)=0$ if, and only if, $x=y$, and (ii) $d(x, y)=d(y, x)$. Let $d$ be a symmetric on a set $X$ and for $r$ $>0$ and any $x \in X$, let $B(x, r)=\{y \in X: d(x, y)<r\}$. A topology $\tau_{d}$ on $X$ is given by $U \in \tau_{d}$ if, and only if, for each $\mathrm{x} \in \mathrm{U}, \mathrm{B}(\mathrm{x}, \mathrm{r}) \subset \mathrm{U}$ for some $\mathrm{r}>0$. A symmetric $\mathrm{d}$ is a semi-metric if for each $\mathrm{x} \in \mathrm{X}$ and each $\mathrm{r}>0, \mathrm{~B}(\mathrm{x}, \mathrm{r})$ is a neighbourhood of $\mathrm{x}$ in the topology $\tau_{\mathrm{d}}$. Note that $\lim _{n \rightarrow \infty} d\left(x_{n}, x\right)=0$ if and only if $\mathrm{x}_{\mathrm{n}} \rightarrow \mathrm{x}$ in the topology $\tau_{\mathrm{d}}$.

\section{Preliminaries}

Before proving our results, we need the following definitions and known results in this sequel.

Definition 2.1([4]) let (X, d) be a symmetric space. (W.3) Given $\left\{\mathrm{x}_{\mathrm{n}}\right\}, \mathrm{x}$ and $\mathrm{y}$ in $\mathrm{X}, \lim _{n \rightarrow \infty} d\left(x_{n}, x\right)=0$ and $\lim _{n \rightarrow \infty} d\left(x_{n}, y\right)=0$ imply $\mathrm{x}=\mathrm{y}$. (W.4) Given $\left\{\mathrm{x}_{\mathrm{n}}\right\},\left\{\mathrm{y}_{\mathrm{n}}\right\}$ and $\mathrm{x}$ in $\mathrm{X} \lim _{n \rightarrow \infty} d\left(x_{n}, x\right)=0$ and $\lim _{n \rightarrow \infty} d\left(x_{n}, y_{n}\right)$ $=0$ imply that $\lim _{n \rightarrow \infty} d\left(y_{n}, x\right)=0$.

Definition 2.2 ([12]) Two self mappings A and B of a metric space (X, d) are said to be weakly commuting if $\mathrm{d}(\mathrm{ABx}, \mathrm{BAx}) \leq \mathrm{d}(\mathrm{Ax}, \mathrm{Bx}), \forall \mathrm{x} \in \mathrm{X}$.

Definition 2.3([6]) Let A and B be two self mappings of a metric space $(X, d)$. A and B are said to be compatible if $\lim _{n \rightarrow \infty} d\left(A B x_{n}, B A x_{n}\right)=0$, whenever $\left(\mathrm{x}_{\mathrm{n}}\right)$ is a sequence in $\mathrm{X}$ such that $\lim _{n \rightarrow \infty} A x_{n}=\lim _{n \rightarrow \infty} B x_{n}=\mathrm{t}$ for some $\mathrm{t} \in \mathrm{X}$.

Remark 2.4. Two weakly commuting mappings are compatibles but the converse is not true as is shown in [6].

Definition 2.5 ([7]) Two self mapping $T$ and $S$ of a metric space $X$ are said to be weakly Compatible if they commute at there coincidence points, i.e., if $\mathrm{Tu}=\mathrm{Su}$ for some $\mathrm{u} \in \mathrm{X}$, then $\mathrm{TSu}=\mathrm{STu}$.

Note 2.6. Two compatible maps are weakly compatible. M. Aamri [2] introduced the concept property (E.A) in the following way.

Definition 2.7 ([2]). Let $\mathrm{S}$ and $\mathrm{T}$ be two self mappings of a metric space (X, d). We say that $\mathrm{T}$ and $\mathrm{S}$ satisfy the property (E.A) if there exists a sequence $\left\{\mathrm{x}_{n}\right\}$ such that $\lim _{n \rightarrow \infty} T x_{n}=\lim _{n \rightarrow \infty} S x_{n}=\mathrm{t}$ for some $\mathrm{t} \in \mathrm{X}$.

Definition 2.8 ([2]). Two self mappings $S$ and $T$ of a metric space (X, d) will be non-compatible if there exists at least one sequence $\left\{\mathrm{x}_{\mathrm{n}}\right\}$ in $\mathrm{X}$ such that if $\lim _{n \rightarrow \infty} d\left(S T x_{n}, T S x_{n}\right)$ is either nonzero or non-existent.

Remark 2.9. Two noncompatible self mappings of a metric space (X, d) satisfy the property (E.A).In the sequel, we need a function $\phi: \mathrm{R}^{+} \rightarrow \mathrm{R}^{+}$satisfying the condition $0<\phi(\mathrm{t})<\mathrm{t}$ for each $\mathrm{t}>0$.

Definition 2.10. Let $A$ and $B$ be two self mappings of a symmetric space (X, d). A and B are said to be compatible if $\lim _{n \rightarrow \infty} d\left(A B x_{n}, B A x_{n}\right)=0$ whenever $\left(\mathrm{x}_{\mathrm{n}}\right)$ is a sequence in $\mathrm{X}$ such that $\lim _{n \rightarrow \infty} d\left(A x_{n}, t\right)$ $=\lim _{n \rightarrow \infty} d\left(B x_{n}, t\right)=0$ for some $\mathrm{t} \in \mathrm{X}$.

Definition 2.11. Two self mappings A and B of a symmetric space (X,d) are said to be weakly compatible if they commute at their coincidence points.

Definition 2.12. Let A and B be two self mappings of a symmetric space $(X, d)$.We say that A and B satisfy the property (E.A) if there exists a sequence $\left(\mathrm{x}_{\mathrm{n}}\right)$ such that $\lim _{n \rightarrow \infty} d\left(A x_{n}, t\right)=\lim _{n \rightarrow \infty} d\left(B x_{n}, t\right)=0$ for some $\mathrm{t} \in \mathrm{X}$. 
Remark 2.13. It is clear from the above Definition 2.10, that two self mappings $\mathrm{S}$ and $\mathrm{T}$ of a symmetric space $(\mathrm{X}, \mathrm{d})$ will be noncompatible if there exists at least one sequence $\left(\mathrm{x}_{\mathrm{n}}\right)$ in $\mathrm{X}$ such that $\lim _{n \rightarrow \infty} d\left(S x_{n}, t\right)$ $=\lim _{n \rightarrow \infty} d\left(T x_{n}, t\right)=0$ for some $\mathrm{t} \in \mathrm{X}$. but $\lim _{n \rightarrow \infty} d\left(S T x_{n}, T S x_{n}\right)$ is either non-zero or does not exist. Therefore, two noncompatible self mappings of a symmetric space (X, d) satisfy the property (E.A).

Definition 2.14. Let $(X, d)$ be a symmetric space. We say that $(X, d)$ satisfies the property $\left(H_{E}\right)$ if given $\left\{x_{n}\right\}$, $\left\{\mathrm{y}_{\mathrm{n}}\right\}$ and $\mathrm{x}$ in $\mathrm{X}$, and $\lim _{n \rightarrow \infty} d\left(x_{n}, x\right)=0$ and $\lim _{n \rightarrow \infty} d\left(y_{n}, x\right)=0$ implylim $\operatorname{im}_{n \rightarrow \infty} d\left(y_{n}, x_{n}\right)=0$.

Note that $(X, d)$ is not a metric space.

\section{Implicit Relation}

Implicit relations on metric spaces have been used in many articles. (See [4], [10], [13].

Let $R_{+}$denote the non-negative real numbers and let $\mathrm{F}$ be the set of all Continuous functions $\mathrm{F}: \mathrm{R}_{+} \rightarrow \mathrm{R}_{+}$ satisfying the following conditions:

$\mathbf{F}_{1}$ : there exists an upper semi-continuous and non-decreasing function $\mathrm{f}: \mathrm{R}_{+} \rightarrow \mathrm{R}_{+}$, f $(0)=0$, $\mathrm{f}(t)<t$ for $t>0$, such that for $\mathrm{u} \geq 0, \mathrm{~F}(\mathrm{u}, \mathrm{v}, \mathrm{v}, 0) \leq 0$ or $\mathrm{F}(\mathrm{u}, \mathrm{v}, 0, \mathrm{v}) \leq 0$ or $\mathrm{F}(\mathrm{u}, 0, \mathrm{v}, \mathrm{v}) \leq 0$ implies $\mathrm{u} \leq \mathrm{f}(\mathrm{v})$.

$\boldsymbol{F}_{2}: \mathrm{F}(\mathrm{u}, 0,0,0)>0$ and $\mathrm{F}(\mathrm{u}, \mathrm{u}, \mathrm{u}, 0)>0, \forall \mathrm{u}>0$.

Example (3.1). $F\left(t_{1}, t_{2}, t_{3}, t_{4}\right)=t_{1}-\alpha \max \left\{t_{2}, t_{3}, t_{4}\right\}$, where $0<\alpha<1$.

$\mathrm{F}_{1}$ : Let $\mathrm{u}>0$ and $\mathrm{F}(\mathrm{u}, \mathrm{v}, \mathrm{v}, 0)=\mathrm{u}-\alpha \mathrm{v} \leq 0$, then $\mathrm{u} \leq \alpha \mathrm{v}$. Similarly, let $\mathrm{u}>0$ and $\mathrm{F}(\mathrm{u}, \mathrm{v}, 0, \mathrm{v}) \leq 0$, then $\mathrm{u} \leq \alpha \mathrm{v}$ and again let $\mathrm{u}>0$ and $\mathrm{F}(\mathrm{u}, 0, \mathrm{v}, \mathrm{v}) \leq 0$, then $\mathrm{u} \leq \alpha \mathrm{v}$. If $\mathrm{u}=0$ then $\mathrm{u} \leq \alpha \mathrm{v}$. Thus $\mathrm{F}_{1}$ is satisfied with $\mathrm{f}(\mathrm{t})=\alpha \mathrm{t}$.

$\mathrm{F}_{2}: \mathrm{F}(\mathrm{u}, 0,0,0)=\mathrm{u}>0, \forall \mathrm{u}>0$ and $\mathrm{F}(\mathrm{u}, \mathrm{u}, \mathrm{u}, 0)=\mathrm{u}(1-\alpha)>0, \forall \mathrm{u}>0$.

Thus $\mathrm{F} \in \mathcal{F}$

Example (3.2). $\mathrm{F}\left(\mathrm{t}_{1}, \mathrm{t}_{2}, \mathrm{t}_{3}, \mathrm{t}_{4}\right)=t_{l}-\psi\left(\max \left\{\mathrm{t}_{2}, \mathrm{t}_{3}, \mathrm{t}_{4}\right\}\right)$, where: $\mathrm{R}_{+} \rightarrow \mathrm{R}_{+}$is upper semi-continuous, nondecreasing and $\psi(0)=0, \psi(\mathrm{t})<\mathrm{t}$ for $\mathrm{t}>0$.

$\mathbf{F}_{1}$ : Let $\mathrm{u}>0$ and $\mathrm{F}(\mathrm{u}, \mathrm{v}, \mathrm{v}, 0)=\mathrm{u}-\psi(\mathrm{v}) \leq 0$, then $\mathrm{u} \leq \psi(\mathrm{v})$. Similarly, let

$\mathrm{u}>0$ and $\mathrm{F}(\mathrm{u}, \mathrm{v}, 0, \mathrm{v}) \leq 0$, then $\mathrm{u} \leq \psi(\mathrm{v})$ and again let $\mathrm{u}>0$ and $\mathrm{F}(\mathrm{u}, 0, \mathrm{v}, \mathrm{v}) \leq 0$,

then $u \leq \psi(v)$. If $u=0$ then $u \leq \psi(v)$. Thus $\mathrm{F}_{1}$ is satisfied with $\mathrm{f}=\psi$.

$\mathbf{F}_{2}: \mathrm{F}(\mathrm{u}, 0,0,0)=\mathrm{u}>0, \forall \mathrm{u}>0$ and $\mathrm{F}(\mathrm{u}, \mathrm{u}, \mathrm{u}, 0)=\mathrm{u}-\psi(\mathrm{u})>0, \forall \mathrm{u}>0$.

Thus $\mathrm{F} \in \mathcal{F}$

\section{IV.Main Result}

Theorem 4.1: Let $d$ be a symmetric for $X$ that satisfies (W.3), (W.4) and ( $\left.H_{E}\right)$. Let $\left\{A_{i}\right\},\left\{A_{j}\right\}(i \neq j)$ and $S$ be self mappings of $(\mathrm{X}, \mathrm{d})$ such that

(1) $\mathrm{F}\left(\int_{0}^{d\left(A_{i} x, A_{j} y\right)} \phi(t) d t, \int_{0}^{d(S x, S y)} \phi(t) d t, \int_{0}^{d\left(S x, A_{j} y\right)} \phi(t) d t, \int_{0}^{d\left(S y, A_{j} y\right)} \phi(t) d t\right) \leq 0$.

for all $(\mathrm{x}, \mathrm{y}) \in \mathrm{X}^{2},(\mathrm{i} \neq \mathrm{j})$ where $\mathrm{F} \in \mathcal{F}$ and $\phi: \mathrm{R}_{+} \rightarrow \mathrm{R}_{+}$is a Lebesque-integrable mapping which is summable, non-negative and such that (2) $\int_{0}^{\varepsilon} \phi(t) d t>0$ for all $\varepsilon>0$.

Suppose that $A_{i} X \subset S X$ and $A_{j} X \subset S X,(i \neq j)\left(A_{i}, S\right)$ and $\left(A_{j}, S\right)(i \neq j)$ are weakly compatible and $\left(A_{i}, S\right)$ or $\left(A_{j}, S\right)$ $(i \neq j)$ satisfies property (E.A). If the range of one ofthe mappings $\left\{A_{i}\right\},\left\{A_{j}\right\}$ or $S(i \neq j)$ is a closed subspace of $X$, then $\left\{A_{i}\right\},\left\{A_{j}\right\}$ and $S(i \neq j)$ have a unique common fixed point in $X$.

Proof: Suppose that $\left\{\mathrm{A}_{\mathrm{j}}\right\}$ and $T, \forall \mathrm{j}$ satisfy property $(E . A)$. Then, there exists a sequence $\left\{x_{\mathrm{n}}\right\}$ in $X$ such that that $\lim _{n \rightarrow \infty} d\left(A_{j} x_{n}, z\right)=\lim _{n \rightarrow \infty} d\left(S x_{n}, z\right)=0$ for some $\mathrm{z} \in \mathrm{X} . \forall \mathrm{j}$

Therefore, by $\left(\mathrm{H}_{\mathrm{E}}\right)$ we have $\lim _{n \rightarrow \infty} d\left(A_{j} x_{n}, S x_{n}\right)=0 . \forall \mathrm{j}$

Since $\mathrm{A}_{\mathrm{j}}(X) \subset S(X) \forall \mathrm{j}$, there exists in $X$ a sequence $\left\{y_{n}\right\}$ such that $\mathrm{A}_{\mathrm{j}} \mathrm{x}_{\mathrm{n}}=\mathrm{Sy}_{\mathrm{n}} . \forall \mathrm{j}$

Hence, $\lim _{n \rightarrow \infty} d\left(S y_{n}, z\right)=0$.

Let us show that $\lim _{n \rightarrow \infty} d\left(A_{i} y_{n}, z\right)=0 . \forall \mathrm{i}$.

Suppose that $\operatorname{im}_{n \rightarrow \infty} d\left(A_{i} y_{n}, A_{j} x_{n}\right)>0$. Then, using (1), we have

$\mathrm{F}\left(\int_{0}^{d\left(A_{i} y_{n}, A_{j} x_{n}\right)} \phi(t) d t, \int_{0}^{d\left(S y_{n}, S x_{n}\right)} \phi(t) d t, \int_{0}^{d\left(S y_{n}, A_{j} x_{n}\right)} \phi(t) d t, \int_{0}^{d\left(S x_{n}, A_{j} x_{n}\right)} \phi(t) d t\right) \leq 0 .(\mathrm{i} \neq \mathrm{j})$

We have,

$\mathrm{F}\left(\lim _{n \rightarrow \infty} \int_{0}^{d\left(A_{i} y_{n}, A_{j} x_{n}\right)} \phi(t) d t, \lim _{n \rightarrow \infty} \int_{0}^{d\left(A_{j} x_{n}, S x_{n}\right)} \phi(t) d t, \lim _{n \rightarrow \infty} \int_{0}^{d\left(A_{j} x_{n}, S x_{n}\right)} \phi(t) d t,\right) \leq 0$.

$(\mathrm{i} \neq \mathrm{j})$. From $F_{1}$, there exists an upper semi-continuous and non-decreasing function $f: \mathrm{R}_{+} \rightarrow \mathrm{R}_{+} f(0)=0, f(t)<t$ for $t>0$

such that $\lim _{n \rightarrow \infty} \int_{0}^{d\left(A_{i} y_{n}, A_{j} x_{n}\right)} \phi(\mathrm{t}) \mathrm{dt} \leq \mathrm{f}\left(\lim _{n \rightarrow \infty} \int_{0}^{d\left(A_{j} x_{n}, S x_{n}\right)} \phi(t) d t\right)<\lim _{n \rightarrow \infty} \int_{0}^{d\left(A_{j} x_{n}, S x_{n}\right)} \phi(t) d t,(\mathrm{i} \neq \mathrm{j})$.

Therefore $\lim _{n \rightarrow \infty} \int_{0}^{d\left(A_{j} x_{n}, S x_{n}\right)} \phi(t) d t,>0$ which is a contradiction. Then we have that $\lim _{n \rightarrow \infty} \int_{0}^{d\left(A_{i} y_{n}, A_{j} x_{n}\right)} \phi(t) d t=0$. By $(W .4)$, we deduce that $\lim _{n \rightarrow \infty} d\left(A_{i} y_{n}, z\right)=0 . \forall \mathrm{i}$ 
Suppose that $S X$ is a closed subspace of $X$. Then $z=\mathrm{Su}$ for some $\mathrm{u} \in \mathrm{X}$. Consequently, we have $\lim _{n \rightarrow \infty} d\left(A_{i} y_{n}, A_{j} x_{n}\right)=\lim _{n \rightarrow \infty} d\left(A_{j} x_{n}, S u\right)=\lim _{n \rightarrow \infty} d\left(S x_{n}, \mathrm{Su}\right)=\lim _{n \rightarrow \infty} d\left(S y_{n}, \mathrm{Su}\right)=0$.

We claim that $\mathrm{Au}=S \mathrm{u}$. Using (1),

$\mathrm{F}\left(\int_{0}^{d\left(A_{i} u, A_{j} x_{n}\right)} \phi(t) d t, \int_{0}^{d\left(\mathrm{Su}, S x_{n}\right)} \phi(t) d t, \int_{0}^{d\left(S u_{,}, A_{j} x_{n}\right)} \phi(t) d t, \int_{0}^{d\left(S x_{n} A_{j} x_{n}\right)} \phi(t) d t,\right) \leq 0$.

and letting $n \rightarrow \infty$, we have $\mathrm{F}\left(\int_{0}^{d\left(A_{i} u, A_{j} x_{n}\right)} \emptyset(t) d t, 0,0,0\right) \leq 0 . \forall \mathrm{i}, \mathrm{j}(\mathrm{i} \neq \mathrm{j})$.

which is a contradiction with $F_{2}$, if $\lim _{n \rightarrow \infty} \int_{0}^{d\left(A_{i} u, A_{j} x_{n}\right)} \phi(\mathrm{t}) \mathrm{dt}>0$

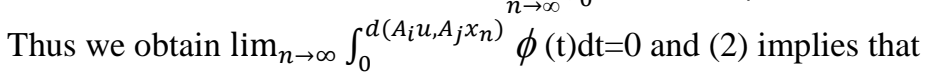

$\lim _{n \rightarrow \infty} d\left(A_{i} u, A_{j} x_{n}\right)=0(\mathrm{i} \neq \mathrm{j})$.

By (W.3) we have $z=\mathrm{A}_{\mathrm{i}} \mathrm{u}=\mathrm{Su} . \forall \mathrm{i}$ The weak compatibility of $\left\{A_{\mathrm{i}}\right\}$ and $S \forall_{\mathrm{i}}$ implies that

$\mathrm{A}_{\mathrm{i}} \mathrm{Su}=\mathrm{SA} \mathrm{u} \forall$ i ; i.e., $\mathrm{A}_{\mathrm{i}} \mathrm{Z}=\mathrm{Sz} . \forall \mathrm{i}$ On the other hand, since $\mathrm{A}_{\mathrm{i}} \mathrm{X} \subseteq \mathrm{SX}, \forall$ i there exists

$\mathrm{v} \in \mathrm{X}$ such that $\mathrm{A}_{\mathrm{i}} \mathrm{u}=\mathrm{Sv} . \forall \mathrm{i}$ We claim that $\mathrm{A}_{\mathrm{j}} \mathrm{v}=\mathrm{Sv} . \forall \mathrm{j}$ If not, condition (1) gives

$\mathrm{F}\left(\int_{0}^{d\left(A_{i} u, A_{j} v\right)} \phi(t) d t, \int_{0}^{d(S u, S v)} \phi(t) d t, \int_{0}^{d\left(S u, A_{j} v\right)} \phi(t) d t, \int_{0}^{d\left(S u, A_{j} v\right)} \phi(t) d t\right) \leq 0 .(\mathrm{i} \neq \mathrm{j})$.

And we have, $\mathrm{F}\left(\int_{0}^{d\left(A_{i} u, A_{j} v\right)} \phi(t) d t, \int_{0}^{d\left(S v, A_{j} v\right)} \phi(t) d t, \int_{0}^{d\left(S v, A_{j} v\right)} \phi(t) d t,\right) \leq 0$. (i $\left.\neq \mathrm{j}\right)$.

From $\mathrm{F}_{2}, \int_{0}^{d\left(S u, A_{j} v\right)} \phi(t) d t=\int_{0}^{d\left(A_{i} u, A_{j} v\right)} \phi(t) d t \leq f\left(\int_{0}^{d\left(S v, A_{j} v\right)} \phi(t) d t\right)(\mathrm{i} \neq \mathrm{j})$.

Which is a contradiction since $\int_{0}^{d\left(S v, A_{j} v\right)} \phi(t) d t>0$, by (2)

Hence, $\mathrm{z}=\mathrm{A}_{\mathrm{i}} \mathrm{u}=\mathrm{Su}=\mathrm{A}_{\mathrm{j}} \mathrm{v}=\mathrm{Sv}$. $(\mathrm{i} \neq \mathrm{j})$, the weak compatibility of $\left\{\mathrm{A}_{\mathrm{j}}\right\}$ and $\mathrm{S} \forall \mathrm{j}$ implies that $\mathrm{A}_{\mathrm{j}} \mathrm{Sv}=\mathrm{SA} \mathrm{A}_{\mathrm{j}} \mathrm{v}$

i.e., $A_{j} z=S z$.

Let us show that $z$ is a common fixed point of $\left\{A_{i}\right\},\left\{A_{j}\right\}$, and $S(i \neq j)$.

If $\mathrm{z} \neq \mathrm{A}_{\mathrm{i}} \mathrm{z}, \forall \mathrm{i}$ using (1), we get

$\mathrm{F}\left(\int_{0}^{d\left(A_{i}, A_{j} v\right)} \phi(t) d t, \int_{0}^{d(S z, S v)} \phi(t) d t, \int_{0}^{d\left(S z, A_{j} v\right)} \phi(t) d t, \int_{0}^{d\left(S z, A_{j} v\right)} \phi(t) d t,\right) \leq 0 .(\mathrm{i} \neq \mathrm{j})$

And we have, $\mathrm{F}\left(\int_{0}^{d\left(A_{i} z, z\right)} \phi(t) d t, \int_{0}^{d\left(A_{i} z, z\right)} \phi(t) d t, \int_{0}^{d\left(A_{i} z, z\right)} \phi(t) d t, 0\right) \leq 0$. (i $\left.\neq \mathrm{j}\right)$

Which is a contradiction with $\mathrm{F}_{2}$, since $\int_{0}^{d\left(A_{i} z, z\right)} \phi(\mathrm{t}) \mathrm{dt}>0$ by (2)

Thus $\mathrm{z}=\mathrm{A}_{\mathrm{i}} \mathrm{z}=\mathrm{Sz} \forall \mathrm{i}$

If $\mathrm{z} \neq \mathrm{A}_{\mathrm{j}} \mathrm{z}$ using (1) we get

$\mathrm{F}\left(\int_{0}^{d\left(A_{i} z, A_{j} z\right)} \phi(t) d t, \int_{0}^{d(S z, S z)} \phi(t) d t, \int_{0}^{d\left(S z, A_{j} z\right)} \phi(t) d t, \int_{0}^{d\left(S z, A_{j} z\right)} \phi(t) d t,\right) \leq 0 .(\mathrm{i} \neq \mathrm{j})$

And we have $\mathrm{F}\left(\int_{0}^{d\left(z, A_{j} z\right)} \phi(t) d t, 0, \int_{0}^{d\left(z, A_{j} z\right)} \phi(t) d t, \int_{0}^{d\left(z, A_{j} z\right)} \phi(t) d t,\right) \leq 0 .(\mathrm{i} \neq \mathrm{j})$

which is a contradiction with $F_{2}$ since $\int_{0}^{d\left(z, A_{j} Z\right)} \phi(t) d t>0$ by (2).

Thus $z=\mathrm{A}_{\mathrm{j}} \mathrm{z}=\mathrm{Sz}=\mathrm{A}_{\mathrm{i}} \mathrm{z}$.

The cases in which $A_{\mathrm{i}} X$ or $\mathrm{A}_{\mathrm{j}} \mathrm{X}$ is a closed subspace of $X$ are similar to the cases in which $S X$ is closed since $\mathrm{A}_{\mathrm{i}} \mathrm{X} \subset \mathrm{SX}$ and $\mathrm{A}_{\mathrm{j}} \mathrm{X} \subset \mathrm{SX}$.(i $\left.\neq \mathrm{j}\right)$

Uniqueness.

For the uniqueness of $z$, suppose that $w \neq \mathrm{z}$ is another common fixed point of $\left\{\mathrm{A}_{\mathrm{i}}\right\},\left\{\mathrm{A}_{\mathrm{j}}\right\}$ and $\mathrm{S}$. (i $\mathrm{i} \neq \mathrm{j}$ )

Using (1), we obtain, $\mathrm{F}\left(\int_{0}^{d\left(A_{i} z, A_{j} w\right)} \phi(t) d t, \int_{0}^{d(S z, S w)} \phi(t) d t, \int_{0}^{d\left(S z, A_{j} w\right)} \phi(t) d t, \int_{0}^{d\left(S w, A_{j} w\right)} \phi(t) d t\right.$, $) \leq 0$. (i $\left.\neq \mathrm{j}\right)$

And we have, $\mathrm{F}\left(\int_{0}^{d(z, w)} \phi(t) d t, \int_{0}^{d(z, w)} \phi(t) d t, \int_{0}^{d(z, w)} \phi(t) d t, 0\right) \leq 0 .(\mathrm{i} \neq \mathrm{j})$

which is a contradiction with $\mathrm{F}_{2}$ since $\int_{0}^{d(z, w)} \phi(t) d t>0$ by (2). Thus $z=w$, and the common fixed point is unique. This completes the proof of the theorem.

Corollary4.2: Let $d$ be a symmetric for $X$ that satisfies (W.3),(W.4) and $\left(\mathrm{H}_{\mathrm{E}}\right)$.Let A,B and $\mathrm{S}$ be self mappings of $(\mathrm{X}, \mathrm{d})$ such that (1)F $\left(\int_{0}^{d(A x, B y)} \phi(t) d t, \int_{0}^{d(S x, S y)} \phi(t) d t, \int_{0}^{d(S x, B y)} \phi(t) d t, \int_{0}^{d(S y, B y)} \phi(t) d t,\right) \leq 0$.

for all $(\mathrm{x}, \mathrm{y}) \in \mathrm{X}^{2}$, where $\mathrm{F} \in \mathcal{F}$ and $\phi: \mathrm{R}_{+} \rightarrow \mathrm{R}_{+}$is a Lebesque-integrable mapping which is summable, nonnegative and such that (2) $\int_{0}^{\varepsilon} \phi(t) d t>0$ for all $\varepsilon>0$.

Suppose that $\mathrm{AX} \subset \mathrm{SX}$ and $\mathrm{BX} \subset \mathrm{SX},(\mathrm{A}, \mathrm{S})$ and $(\mathrm{B}, \mathrm{S})$ are weakly compatible and $(\mathrm{A}, \mathrm{S})$ or $(\mathrm{B}, \mathrm{S})$ satisfies property (E.A). If the range of one of the mappings $A, B$ or $S$ is a closed subspace of $X$, then $A, B$ and $S$ have a unique common fixed point in $\mathrm{X}$.

Proof. The proof of Corollary 4.2 follows from theorem 4.1 by putting $A_{i}=A ; A_{j}=B$. $(i \neq j)$. 
Theorem4.3. Let $d$ be a symmetric for $X$ that satisfies (W.3),(W.4) and $\left(\mathrm{H}_{\mathrm{E}}\right)$.Let $\left\{\mathrm{A}_{\mathrm{i}}\right\}, \mathrm{B} \forall \mathrm{i}$ be self mappings of (X,d) such that (1)F $\mathrm{F}\left(\int_{0}^{d\left(A_{i} x, A_{i} y\right)} \phi(t) d t, \int_{0}^{d(B x, B y)} \phi(t) d t, \int_{0}^{d\left(B x, A_{i} y\right)} \phi(t) d t, \int_{0}^{d\left(A_{i}, y, B y\right)} \phi(t) d t,\right) \leq 0 . \forall \mathrm{i}$

for all $(\mathrm{x}, \mathrm{y}) \in \mathrm{X}^{2}$, where $\mathrm{F} \in \mathcal{F}$ and $\phi: \mathrm{R}_{+} \rightarrow \mathrm{R}_{+}$is a Lebesque-integrable mapping which is summable, nonnegative and such that (2) $\int_{0}^{\varepsilon} \phi(t) d t>0$ for all $\varepsilon>0$. Suppose that $\mathrm{A}_{\mathrm{i}} \mathrm{X} \subset \mathrm{BX} \forall \mathrm{i},\left(\mathrm{A}_{\mathrm{i}}, \mathrm{B}\right) \forall \mathrm{i}$ is weakly compatible and $\left(\mathrm{A}_{\mathrm{i}}, \mathrm{B}\right) \forall \mathrm{i}$ satisfy the property (E.A). If the range of one of the mappings $\left\{\mathrm{A}_{\mathrm{i}}\right\}$, or $B$ is a closed subspace of $X$, then $\left\{A_{i}\right\}$ and $B \quad \forall$ i have a unique common fixed point in $X$.

Proof. Suppose that $\mathrm{A}_{\mathrm{j}}$ and $\mathrm{B} \forall \mathrm{j}$ satisfy property (E.A). Then there exists a sequence $\left\{x_{\mathrm{n}}\right\}$ in $X$ such that that $\lim _{n \rightarrow \infty} d\left(A_{i} x_{n}, z\right)=\lim _{n \rightarrow \infty} d\left(B x_{n}, z\right)=0$ for some $\mathrm{z} \in \mathrm{X}$.

Therefore, by $\left(\mathrm{H}_{\mathrm{E}}\right)$ we have $\lim _{n \rightarrow \infty} d\left(A_{i} x_{n}, B x_{n}\right)=0 . \forall$ i.

Since $\mathrm{A}_{\mathrm{i}} X \subset \mathrm{B} X \forall \mathrm{i}$, there exists in $X$ a sequence $\left\{y_{n}\right\}$ such that $\mathrm{A}_{\mathrm{i}} \mathrm{x}_{\mathrm{n}}=\mathrm{By}$.

Hence, $\lim _{n \rightarrow \infty} d\left(B y_{n}, z\right)=0$.

Let us show that $\lim _{n \rightarrow \infty} d\left(A_{i} y_{n}, z\right)=0 . \forall \mathrm{i}$.

Suppose that $\lim _{n \rightarrow \infty} d\left(A_{i} y_{n}, A_{i} x_{n}\right)>0$. Then, using (1), we have

$\mathrm{F}\left(\int_{0}^{d\left(A_{i} y_{n}, A_{i} x_{n}\right)} \phi(t) d t, \int_{0}^{d\left(B y_{n}, B x_{n}\right)} \phi(t) d t, \int_{0}^{d\left(B y_{n}, A_{i} x_{n}\right)} \phi(t) d t, \int_{0}^{d\left(, A_{i} x_{n}, B x_{n}\right),} \phi(t) d t,\right) \leq 0 . \forall \mathrm{i}$

And we have, $F\left(\lim _{n \rightarrow \infty} \int_{0}^{d\left(A_{i} y_{n}, A_{i} x_{n}\right)} \phi(t) d t, \lim _{n \rightarrow \infty} \int_{0}^{d\left(A_{i} x_{n}, B x_{n}\right)} \phi(t) d t, 0, \lim _{n \rightarrow \infty} \int_{0}^{d\left(A_{i} x_{n}, B x_{n}\right)} \phi(t) d t,\right) \leq$

0. $\forall \mathrm{i}$ From $F_{1}$, there exists an upper semi-continuous and non-decreasing function

$f: \mathrm{R}_{+} \rightarrow \mathrm{R}_{+} f(0)=0, f(t)<t$ for $t>0$ such that $\lim _{n \rightarrow \infty} \int_{0}^{d\left(A_{i} y_{n}, A_{i} x_{n}\right)} \phi(\mathrm{t}) \mathrm{dt} \leq$

$\mathrm{f}\left(\lim _{n \rightarrow \infty} \int_{0}^{d\left(A_{i} x_{n}, B x_{n}\right)} \phi(t) d t\right)<\lim _{n \rightarrow \infty} \int_{0}^{d\left(A_{i} x_{n}, B x_{n}\right)} \phi(t) d t,(\mathrm{i} \neq \mathrm{j})$.

Therefore $\lim _{n \rightarrow \infty} \int_{0}^{d\left(A_{i} x_{n}, B x_{n}\right)} \phi(t) d t,>0$ which is a contradiction. Then we have

that $\lim _{n \rightarrow \infty} \int_{0}^{d\left(A_{i} y_{n}, A_{i} x_{n}\right)} \phi(t) d t=0$. (2) implies thatlim ${ }_{n \rightarrow \infty} d\left(A_{i} y_{n}, A_{i} x_{n}\right)=0$

By (W.4), we deduce that $\lim _{n \rightarrow \infty} d\left(A_{i} y_{n}, z\right)=0 . \forall$ i.

Suppose that $\mathrm{B} X$ is a closed subspace of $X$. Then $z=\mathrm{Bu}$ for some $\mathrm{u} \in \mathrm{X}$. Consequently, we have $\lim _{n \rightarrow \infty} d\left(A_{i} y_{n}, A_{i} x_{n}\right)=\lim _{n \rightarrow \infty} d\left(B x_{n}, B u\right)=\lim _{n \rightarrow \infty} d\left(B y_{n}, B u\right)=\lim _{n \rightarrow \infty} d\left(A_{i} x_{n}, B u\right)=0$. We claim that $\mathrm{Au}$ $=\mathrm{Bu}$. Using (1), $\mathrm{F}\left(\int_{0}^{d\left(A_{i} u, B x_{n}\right)} \phi(t) d t, \int_{0}^{d\left(B u, B x_{n}\right)} \phi(t) d t, \int_{0}^{d\left(B u, A_{i} x_{n}\right)} \phi(t) d t, \int_{0}^{d\left(A_{i} x_{n}, B x_{n}\right)} \phi(t) d t,\right) \leq 0 . \forall \mathrm{i}$

and letting $n \rightarrow \infty$, we have $\mathrm{F}\left(\int_{0}^{d\left(A_{i} u, B x_{n}\right)} \phi(t) d t, 0,0,0\right) \leq 0 . \forall$ i.

which is a contradiction with $F_{2}$, if $\lim _{n \rightarrow \infty} \int_{0}^{d\left(A_{i} u, B x_{n}\right)} \phi(\mathrm{t}) \mathrm{dt}>0$. Thus we obtain $\lim _{n \rightarrow \infty} \int_{0}^{d\left(A_{i} u, B x_{n}\right)} \phi(t) d t=$ 0 and (2) implies that $\lim _{n \rightarrow \infty} d\left(A_{i} u, B x_{n}\right)=0 \forall \mathrm{i}$.

By (W.3) we have $z=\mathrm{A}_{\mathrm{i}} \mathrm{u}=\mathrm{B}_{\mathrm{u}} . \forall \mathrm{i}$ The weak compatibility of $\left\{A_{\mathrm{i}}\right\}$ and $\mathrm{B} \forall \mathrm{i}$, implies that

$\mathrm{A}_{\mathrm{i}} \mathrm{B}_{\mathrm{u}}=\mathrm{BA}_{\mathrm{i}} \mathrm{u} \forall$ i; i.e., $\mathrm{A}_{\mathrm{i}} \mathrm{Z}=\mathrm{Bz} . \forall \mathrm{i}$

The proof is similar when $\mathrm{A}_{\mathrm{i}} \mathrm{X} \forall \mathrm{i}$ is assumed to be a closed subspace of $\mathrm{X}$, since, $\mathrm{A}_{\mathrm{i}} \mathrm{X} \subset \mathrm{BX} \forall \mathrm{i}$

Uniqueness.

If $\mathrm{A}_{\mathrm{i}} \mathrm{u}=\mathrm{Bu}=\mathrm{u}$ and $\mathrm{A}_{\mathrm{i}} \mathrm{v}=\mathrm{Bv}=\mathrm{v} \forall \mathrm{i}$ and $\mathrm{u} \neq \mathrm{v}$ then (1) given,

$\mathrm{F}\left(\int_{0}^{d\left(A_{i} u, A_{i} v\right)} \phi(t) d t, \int_{0}^{d(B u, B v)} \phi(t) d t, \int_{0}^{d\left(B u, A_{i} v\right)} \phi(t) d t, \int_{0}^{d\left(A_{i} v, B v\right)} \phi(t) d t,\right) \leq 0 . \forall \mathrm{i}$

And we have

$\mathrm{F}\left(\int_{0}^{d(u, v)} \phi(t) d t, \int_{0}^{d(u, v)} \phi(t) d t, \int_{0}^{d(u, v)} \phi(t) d t, 0\right) \leq 0 . \forall \mathrm{i}$.

which is a contradiction with $\mathrm{F}_{2}$ since $\int_{0}^{d(u, v)} \emptyset(t) d t>0$ by (2). Thus $\mathrm{u}=\mathrm{v}$ and the common fixed point is unique. This completes the proof of the theorem.

Corollary 4.4: Let $d$ be a symmetric for X that satisfies (W.3),(W.4) and $\left(\mathrm{H}_{\mathrm{E}}\right)$.Let A,B be self mappings of $(\mathrm{X}, \mathrm{d})$ such that $(1) \mathrm{F}\left(\int_{0}^{d(A x, A y)} \phi(t) d t, \int_{0}^{d(B x, B y)} \phi(t) d t, \int_{0}^{d(B x, A y)} \phi(t) d t, \int_{0}^{d(A y, B y)} \phi(t) d t,\right) \leq 0$.

for all $(\mathrm{x}, \mathrm{y}) \in \mathrm{X}^{2}$, where $\mathrm{F} \in \mathcal{F}$ and $\phi: \mathrm{R}_{+} \rightarrow \mathrm{R}_{+}$is a Lebesque-integrable mapping which is summable, nonnegative and such that (2) $\int_{0}^{\varepsilon} \phi(t) d t>0$ for all $\varepsilon>0$.

Suppose that $\mathrm{AX} \subset \mathrm{BX},(\mathrm{A}, \mathrm{B})$ is weakly compatibles and (A, B) satisfy the property (E.A). If the range of one of the mappings $\mathrm{A}$ or $\mathrm{B}$ is a closed subspace of $\mathrm{X}$, then $\mathrm{A}, \mathrm{B}$ have a unique common fixed point in $\mathrm{X}$.

Proof. The proof of Corollary 4.4 follows from theorem 4.3 by putting $\mathrm{A}_{\mathrm{i}}=\mathrm{A} \forall \mathrm{i}$.

If $\phi(t)=1, A_{\mathrm{i}}=\mathrm{A}, \forall \mathrm{i}$ and in Corollary (4.4), we obtain Theorem 2.1 of [1]. 


\section{References.}

[1] M. Aamri and D. El Moutawakil, Common fixed points under contractive conditions in symmetric spaces, Appl. Math. E-Notes 3 (2003), 156-162.

[2] M. Aamri, D. El Moutawakil, Some new common fixed point theorems under strict contractive conditions, J. Math. Anal. Appl. 270 (2002), 181-188.

[3]. Duran turkoglu and ishak altun a common fixed point theorem for weakly compatible mappings in symmetric spaces satisfying an implicit relation bol. soc. mat. mexicana (3) vol. 13, 2007.

[4] I. Altun, H. A. Hancer and D. Turkoglu, A fixed point theorem for multi-maps satisfying an implicit relation on metrically convex metric spaces, Math. Commun. 11 (2006), 17-23.

[5] T. L. Hicks and B. E Rhoades, Fixed point theory in symmetric spaces with applications to probabilistic spaces, Nonlinear Anal., 36 (1999), 331-344.

[6] G. Jungck, Compatible mappings and common fixed points, Int. J. Math. Math. Sci. 9 (1986), 771-779.

[7] G. Jungck, Common fixed points for noncontinuous nonself maps on nonmetric spaces, Far East J. Math. Sci. (4) (2), (1996), 199215.

[8] R. P. Pant, Common fixed points of contractive maps, J. Math. Anal. Appl. 226 (1998), 251-258.

[9] R. P. Pant, Common fixed points of sequences of mappings, Ganita 4 (1996), 43-49.

[10] V. Popa, Some fixed point theorems for compatible mappings satisfying an implicit relation,Demonstratio Math. 32 (1) (1999), 157163.

[11] B. E. Rhoades, Two fixed point theorems for mappings satisfying a general contractive condition of integral type, Int. J. Math. Math. Sci. 2003 (2003), no. 63, 4007-4013.

[12] S. Sessa, on a weak commutativity condition of mappings in fixed point considerations, Publ.Inst. Math. 32 (46), (1982), 149-153.

[13] S. Sharma and B. Desphande, on compatible mappings satisfying an implicit relation in common fixed point consideration, Tamkang J. Math. 33 (3), (2002), 245-252.

[14] W. A. Wilson, On semi-metric spaces, Amer. J. Math. 53 (1931), 361-373. 